\title{
Prospective assessment of hospital-acquired bloosdstream infections: how many may be preventable?
}

\author{
Christine Bonnal, ${ }^{1}$ Bruno Mourvillier, ${ }^{2}$ Régis Bronchard, ${ }^{3}$ Danielle de Paula, ${ }^{1}$ \\ Laurence Armand-Lefevre, ${ }^{4}$ François L'heriteau, ${ }^{1}$ Jean-Luc Quenon, \\ Jean-Christophe Lucet $^{1}$
}

${ }^{1}$ Infection Control Unit Bichat-Claude Bernard University Hospital, Assistance Publique-Hôpitaux de Paris, and Paris VII Denis Diderot University, Paris, France ${ }^{2}$ Medical Intensive Care Unit, Bichat-Claude Bernard University Hospital, Assistance Publique-Hôpitaux de Paris, Paris, France

${ }^{3}$ Surgical Intensive Care Unit, Bichat-Claude Bernard University Hospital, Assistance Publique-Hôpitaux de Paris, Paris, France

${ }^{4}$ Bacteriology Laboratory, Bichat-Claude Bernard University Hospital, Assistance Publique-Hôpitaux de Paris, Paris, France

${ }^{5}$ Comité de Coordination de I'Evaluation Clinique et de la Qualité en Aquitaine, Xavier Arnozan Hospital, Pessac, France

\section{Correspondence to}

Dr Jean-Christophe Lucet, Unité d'Hygiène et de Lutte contre I'Infection Nosocomiale, GH Bichat-Claude Bernard, 75877 Paris Cedex 18, France; jean-christophe.lucet@bch. aphp.fr

Accepted 21 December 2009 Published Online First 27 May 2010

\begin{abstract}
Objective To determine the proportion of preventable hospital-acquired bloodstream infections (HA-BSIs), the authors prospectively examined consecutive cases in a large university hospital over an 18-month period.

Patients and methods Medical charts were assessed with the physician in charge of the patient within 4 days after HA-BSI diagnosis to determine whether the infection was healthcare-related. Preventability was assessed using a validated tool. Results of $378 \mathrm{HA}-\mathrm{BSIs}$ (incidence rate, 1.00 per 1000 patient-days), 341 were first HA-BSI episodes in a patient, and 272 (79.8\%) were secondary to an identifiable source, of whom 196 $(57.5 \%)$ were related to medical management. These $196 \mathrm{HA}-\mathrm{BS}$ ls were related to an invasive procedure $(n=163)$, a non-invasive medical management $(n=30)$ or both $(\mathrm{n}=3)$.
\end{abstract}

Results Of the 272 patients with HA-BSIs from identifiable sources, 55 (20.2\%) had no underlying disease, 115 (42.3\%) had an ultimately fatal underlying disease, $99(36.4 \%)$ had a rapidly fatal disease, and three $(1.1 \%)$ were not evaluated. Of the 196 iatrogenic HA-BSIs, 66 were considered preventable (most of them being related to an intravascular catheter), 84 were of uncertain preventability, and 46 were not preventable. In total, 66 of the $341 \mathrm{HA}-\mathrm{BSI}$ (19.4\%) were considered preventable, and 191 (56.0\%) were not preventable.

Conclusion Although evaluation of the preventability of hospital-associated adverse events has been reported to be difficult and of limited reliability, our simple method may help to identify wards or HA-BSI types that warrant in-depth evaluation.

\section{INTRODUCTION}

Despite steady improvements over the last decades, controlling hospital-acquired infections (HAIs) in hospitals remains challenging. HAI develops in approximately $5 \%$ of inpatients, causing a huge burden of morbidity, mortality and financial cost. ${ }^{1-4}$ Identifying which HAIs can be prevented is essential to the development of HAI-control strategies. Furthermore, increasing media attention to HAIs and rising patient expectations regarding HAI control have helped to place HAIs under intense scrutiny.

Two methods have been used to identify preventable HAIs. ${ }^{1} 5$ One involves measuring nosocomial infection rates during two consecutive observation periods, before and after implementing a prevention programme. The cornerstone study conducted three decades ago using this method suggested that a surveillance programme may prevent $32 \%$ of all nosocomial infections. ${ }^{1}$ According to a 2003 systematic review of the literature that identified 25 interventional studies, most of them based on multimodal interventions in single centres, $10-71 \%$ of HAIs may be preventable, depending on the type of infection and study design. ${ }^{6}$ The other study method involves reviewing medical records. This method is often used to identify hospital adverse events of any origin and assess their preventability. ${ }^{5} 8$ We used this method to prospectively evaluate the preventability of hospital-acquired (HA) bloodstream infections (BSIs) in our hospital over an 18-month period. We selected BSIs for our study because they are fairly common, easy to detect in the bacteriology laboratory, and associated with high morbidity and mortality. $^{2} 9$

\section{METHODS}

This study was carried out at the Bichat-Claude Bernard hospital, a 900-bed university hospital serving as both a primary and a tertiary care centre for adults in Paris, France. It has 27 wards including six intensive care units (ICUs, with 75 beds), a pulmonary transplantation programme and wards for all the main medical and surgical specialities except haematology and neurosurgery. In 2005, about 30000 patients were admitted for 242000 patient-days

Surveillance of HA-BSIs was started at our hospital in 2002. When a patient was found to have a positive blood culture, an infection-control physician visited the clinical unit where the patient was hospitalised. HA-BSI was defined in this study as one or more positive blood cultures unrelated to an infection incubating at hospital admission; in most cases, the blood samples were taken at least $48 \mathrm{~h}$ after admission or were taken in a newly admitted patient with a recent stay in our hospital. Definition of coagulase-negative staphylococci BSIs and BSIs due to other common skin pathogens was derived from a standard definition: either as two or more blood cultures positive for organisms having identical phenotypes, within $48 \mathrm{~h}$, or as one or more positive blood cultures with clinical evidence of sepsis, no other infectious process and specific antibiotic treatment given by the attending physician. ${ }^{10}$ Other blood cultures positive with common skin pathogens were classified as contaminants. 
HA-BSI with an identifiable source was defined as recovery of the same micro-organism from one or more positive blood cultures and from the suspected source (tracheal secretion, urine, surgical site, soft tissue, intravascular catheter (IVC) or other) or with radiological and clinical signs suggestive of a source, according to standard definitions. ${ }^{11}$ All central IVC-related infections were documented by either a positive quantitative tip culture if the IVC was removed ${ }^{12}$ or a significant differential time to positivity between peripheral and central blood cultures if the IVC was maintained. ${ }^{13}$ HA-BSIs associated with peripheral venous catheters were defined as one or more positive blood cultures; presence of at least two of the following signs at the catheter insertion site: pus, swelling, erythema, tenderness, warmth and cord induration; and no other identifiable source. In the absence of an identifiable source of infection, HA-BSI was classified as primary.

All positive blood cultures were reviewed daily during weekdays by an infection-control physician (CB, JCL or FL). When HA-BSI was suspected, the following information was obtained within 4 days after the results of the positive blood culture(s) were available: demographic characteristics, date of admission to the hospital and to the unit where the blood culture(s) were obtained, severity of the underlying disease at hospital admission as reflected by the McCabe and Jackson score, ${ }^{14}$ portal of entry, whether the positive blood culture indicated BSI or contamination, whether the BSI was hospital-acquired, whether antimicrobials were initiated or modified, and the outcome. These data were obtained by interviewing the attending physician, conducting a physical examination of the patient, and reviewing the medical chart and microbiology results.

Starting in January 2005, we determined whether each HABSI case was healthcare-related and whether it could have been prevented. We distinguished between two categories of healthcare-related HA-BSI. One category was HA-BSI secondary to an identifiable source that was related to any of the following invasive procedures: surgery, mechanical ventilation, central or peripheral vascular catheter or indwelling urinary device. The other category was HA-BSI due to non-invasive management. This category included HA-BSI in patients with chemotherapyinduced neutropenia and no identifiable source and HA-BSI related to non-invasive care (eg, HA-BSI secondary to a hospitalacquired bedsore). If several HA-BSI episodes occurred in the same patient during the same hospital stay, only the first episode was included in the study because consecutive episodes in a same patient and same hospital stay are likely to be not independent. In ICU, HA-BSIs were discussed with a same physician (BM or RB). In other units, the investigator collected data with the medical and nursing staff. Preventability was defined as an unintended bacteraemia that was caused by medical management or as a failure to meet the reasonably expected standard of care. Investigators determined the frequency of infectious risk in a defined situation, the degree of emergency, the complexity of the case, the presence of any coexisting conditions, the known compliance with preventive measures in that unit, the extent to which there was a consensus about the correct therapy or diagnosis for a given situation, and whether there had been negligence or a reasonably avoidable error. We used a questionnaire derived from a recent multicentre study ${ }^{8}$ to evaluate the preventability of healthcarerelated HA-BSIs on a six-point Likert scale, where 1 indicated 'almost certainly preventable' and 6 'almost certainly not preventable,' according to accepted criteria. ${ }^{5} 7$ HA-BSIs with a score of 1 or 2 (certainly or probably preventable) were classified as preventable and those with scores of 5 or 6 as not preventable; scores of 3 or 4 were considered to indicate uncertain preventability. One investigator first discussed each case with the clinician in charge of the patient. In a second step, two investigators (JCL, CD or FL) reached agreement about relation to medical management and preventability through discussion. Examples of evaluations are presented in box 1. The study was approved by our Institutional Review Board, which waived the need for patient's informed consent. All forms were anonymised before computer entry. Categorical variables were compared using the $\chi^{2}$ test of Fisher exact test, as appropriate.

\section{Box 1 Examples of evaluation of healthcare-associated}

bloodstream infections

1. An ICU patient who underwent abdominal surgery for a generalised peritonitis due to bowel necrosis developed a HA-BSI from abdominal origin 4 days after initial surgery, due to Escherichia coli, enterococci and anaerobes. This HA$\mathrm{BSI}$ was rated as not related to healthcare management and not preventable (score of 6 on the Likert scale).

2. A patient developed bacteraemic ventilator-associated pneumonia after 10 days in an ICU. From audits and surveillance data, it was known that most recommendations are followed by the ICU staff. This infection was rated as associated with medical management, and probably not preventable (score of 5 on the Likert scale).

3. A patient developed a deep surgical site infection with bacteraemia 15 days after scheduled cardiac surgery (coronary artery bypass grafting with the two thoracic arteries), due to Staphylococcus aureus. He had a history of diabetes and severe obesity. The local recommendation recommends the preoperative use of nasal decontamination in $S$ aureus carriers. The patient did not receive decontamination before surgery despite being an $S$ aureus carrier. This infection was rated healthcare-associated and probably preventable (score of 2 on the Likert scale).

4. An ICU patient was discharged to the rehabilitation unit with a central venous catheter that was no longer useful for medical management. He developed $48 \mathrm{~h}$ later a catheterrelated BSI with septic shock. This BSI was rated healthcareassociated and certainly preventable (score of 1 on the Likert scale).

5. A patient with a peripheral venous catheter (PVC) developed an HA-BSI related to PVC. The PVC associated with the BSI was left in place for 7 days, and the patient had no difficulty with peripheral venous access. This BSI was rated healthcareassociated and certainly preventable (score of 1 on the Likert scale).

6. An older patient with dementia received a PVC and developed an HA-BSI related with PVC that was left in place for 4 days. The patient tore off the catheter dressing several times. This BSI was rated healthcare-associated and possibly not preventable (score of 4 on the Likert scale).

7. A 22-week pregnant woman adequately received ocytocin for a late miscarriage. She developed 2 days later an endometritis with $\mathrm{BSI}$ due to $E$ coli. There was no recommendation for perioperative antimicrobial prophylaxis. This BSI was rated healthcare-associated and probably not preventable (score of 5 on the Likert scale). 


\section{RESULTS}

From 1 January 2005 to 30 June 2006, 1243 episodes of positive blood cultures occurred, of which 865 were classified as BSIs and 378 as HA-BSIs, for an incidence rate of 1.00 per 1000 inpatientdays (figure 1). HA-BSIs had a crude in-hospital death rate of $34.9 \%$ (132/378). These 378 HA-BSIs occurred in 331 patients. In 10 patients, a second HA-BSI occurred during two different hospital stays. These 10 episodes were included in the analysis. The remaining 37 episodes were second $(n=31)$, third $(n=5)$ or fourth $(n=1)$ episode in a same patient during the same hospital stay. These 37 episodes were excluded from further analysis. Of the remaining $341 \mathrm{HA}-\mathrm{BSIs}, 69(20.2 \%)$ were primary and 272 (79.8\%) secondary to an identifiable source.

The sites of the identifiable source in the 272 secondary HABSIs were IVC $(n=67$, including central venous $(n=34)$, peripheral venous $(n=15)$, and other $(n=18))$, urinary $(n=58)$, abdominal or gynaecological $(n=55)$, surgical site $(n=41)$, pulmonary $(n=23)$, soft tissue $(n=16)$ and other $(n=12)$. Bacteraemias secondary to surgical sites were distributed as follows: after abdominal or gynaecological surgery $(n=17)$, after cardiac surgery $(n=9)$, after urological surgery $(n=8)$, after orthopaedic surgery $(n=2)$ and after other surgery $(n=5)$. Of the

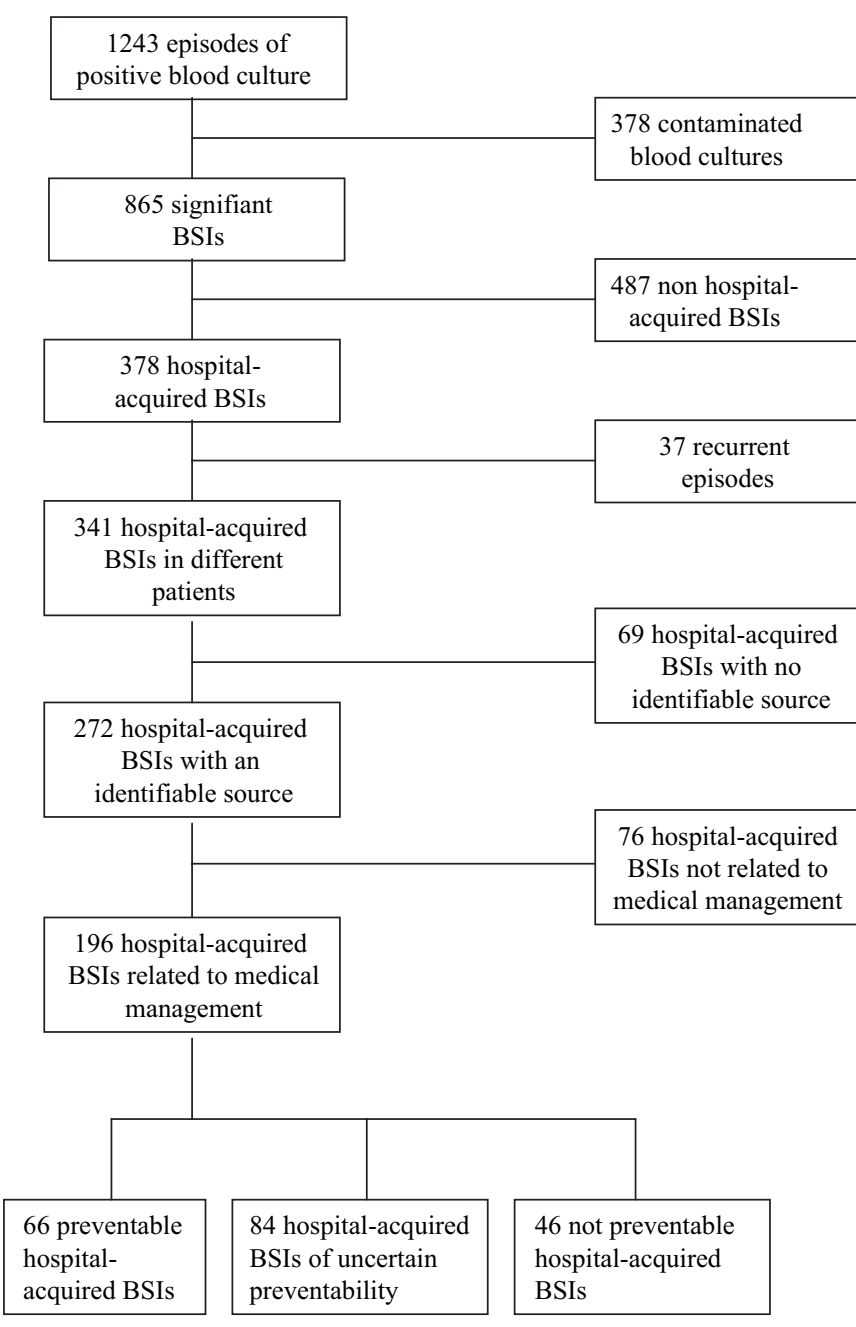

BSI, bloodstream infection

Figure 1 Flow chart of 1243 episodes of positive blood cultures in patients admitted to the Bichat-Claude Bernard Hospital, January 2005-June 2006. BSls, bloodstream infections.
272 episodes with secondary HA-BSI, 55 (20.2\%) had a McCabe score of 0 , indicating no underlying disease, 115 (42.3\%) had a McCabe score of 1 , indicating an ultimately fatal underlying disease, 99 (36.4\%) had a rapidly fatal underlying disease (McCabe score of 2), and three (1.1\%) were not evaluated for this characteristic (table 1). Of the 99 patients with a McCabe score of $2,21(21.2 \%)$ were on palliative care at the time of the HA-BSI. The crude in-hospital death rates in patients with McCabe scores of 0, 1 and 2 were 7/55 (12.7\%), 24/115 (20.9\%) and $51 / 99(51.5 \%)$, respectively. The difference was highly significant $(p<0.0001)$ between death rates in patients with McCabe score of 1 and 2, but not between death rates in patients with McCabe score of 0 and 1 ( $p=0.18$ ). By contrast, older age was not associated with higher mortality $(p>0.25)$ (table 1).

Of the 272 cases of secondary HA-BSI, 196 (72.0\%) were classified as related to medical management: 163 were associated with invasive procedures, 30 with non-invasive procedures, and three with both. Most of the 76 other cases of HA-BSI were classified as stemming from an abdominal $(n=41)$ or urinary $(n=14)$ source (table 2). Of these 196 episodes of HA-BSI related to medical management, $66(33.7 \%)$ were considered preventable, $46(23.4 \%)$ not preventable and $84(42.9 \%)$ of uncertain preventability. IVC-related infections contributed 47 of the 66 preventable HA-BSIs. The 46 unpreventable HA-BSIs were mainly secondary to urinary $(n=12)$, abdominal $(n=11)$ or surgical site $(\mathrm{n}=10)$ infections (table 2$)$.

In total, of the 341 included HA-BSIs, $66(19.4 \%$; $95 \% \mathrm{CI}$ $15.2 \%$ to $23.6 \%)$ were considered preventable. We considered that $191(56.0 \%, 95 \%$ CI $50.7 \%$ to $61.3 \%)$ HA-BSIs were not preventable; they included the 69 primary cases, the 76 secondary HA-BSIs not related to medical management, either invasive procedures or other non-invasive management and the 46 unpreventable secondary HA-BSIs related to medical management. In total, about 45 min were required to assess each HA-BSI case.

\section{DISCUSSION}

These data suggest that $19.4 \%$ of HA-BSIs may be preventable and $56 \%$ unpreventable, whereas $24.6 \%$ were classified of uncertain preventability. To our knowledge, this is the first study that has prospectively assessed the preventability of HA-BSIs. We examined the medical records with the attending physician and examined the patient within a few days after HA-BSI identification, a method that is more accurate than retrospective medical-record review. ${ }^{15}$

Table 1 Severity of underlying disease and outcome in 272 episodes of healthcare-associated bloodstream infection with an identifiable source

\begin{tabular}{lcc}
\hline & $\begin{array}{l}\text { No of cases }(\%) ; \\
\text { total=272 }\end{array}$ & $\begin{array}{l}\text { No of in-hospital } \\
\text { deaths (\%); } \\
\text { total= 83 }\end{array}$ \\
\hline $\begin{array}{l}\text { Male gender }(n(\%)) \\
\text { McCabe score }(\mathrm{n}(\%))\end{array}$ & $164(60)$ & $53 / 164(32.3)$ \\
$\quad$ No underlying disease & $55(20.2)$ & $7 / 55(12.7)$ \\
$\quad$ Ultimately fatal disease & $115(42.3)$ & $24 / 115(20.9)$ \\
$\quad$ Rapidly fatal disease & $99(36.4)$ & $51 / 99(51.5)$ \\
$\quad$ Not evaluated & $3(1.1)$ & $1 / 3(33.3)$ \\
Age (n (\%)) & $101(37.1)$ & $28 / 101(27.7)$ \\
$<60$ years & $124(45.6)$ & $42 / 124(33.9)$ \\
$60-80$ years & $47(17.3)$ & $13 / 47(27.7)$ \\
$>80$ years & & \\
\hline
\end{tabular}


Table 2 Preventability of 196 episodes of hospital-acquired bloodstream infections (HA-BSIs) related to medical management

\begin{tabular}{llllllr}
\hline Source & $\begin{array}{l}\text { Surgical } \\
\text { site }\end{array}$ & Urine & Lung & $\begin{array}{l}\text { Intravascular } \\
\text { catheter }\end{array}$ & $\begin{array}{l}\text { Abdominal or } \\
\text { gynaecological }\end{array}$ & $\begin{array}{l}\text { Total N }= \\
\text { 0ther }\end{array}$ \\
\hline Secondary HA-BSIs & 41 & 58 & 23 & 67 & 55 & 28 \\
HA-BSIs related to medical management & 41 & 44 & 16 & 67 & 14 & $272(79.8)$ \\
Preventable & 6 & 9 & 1 & 47 & 1 & 14 \\
Uncertain preventability & 25 & 23 & 11 & 18 & 2 & 2 \\
Not preventable & 10 & 12 & 4 & 2 & 11 & $56(19.4)$ \\
\hline
\end{tabular}

We focused on HA-BSI for several reasons. First, although HAIs are not the most common adverse events in admitted patients, they are considered important by the general public, and they receive more media attention than other adverse events (eg, drug-related events, bleeding or thrombosis). ${ }^{16}$ That $56 \%$ of HA-BSIs were considered unpreventable in our study is an important finding that deserves to be brought to the attention of both the medical community and the media. Second, as most HA-BSIs are secondary to focal infections, they provide information on a broad range of HAIs. Third, HA-BSIs lead to high morbidity and mortality compared with other HAIs. ${ }^{9}$ Identifying preventable HA-BSIs may help infection-control units and risk-management units to select wards and infection sites that require special attention. Moreover, it is possible to identify preventable cases that may further be evaluated with riskmanagement tools, and be used for awareness and education. Fourth, identification of HA-BSIs cases is easily achieved the bacteriology laboratory and does not require visits to wards, in contrast to other nosocomial infections or non-infectious adverse events. ${ }^{17}$

In our study, $80 \%$ of HA-BSIs were secondary to an identifiable source, in keeping with earlier studies. ${ }^{18} 19$ Because HA-BSI can originate from many sources, it is difficult to compare our results with studies that evaluated preventability by comparing infection rates before and after a multimodal intervention. Most of the earlier studies focused on device-related infections or evaluated all HAIs. ${ }^{6}$ Nevertheless, recent studies of interventions to prevent nosocomial infections found similar results, with about $20-25 \%$ of HAIs being prevented by the interventions. $^{20-23}$

Intravascular catheter-related HA-BSIs may be the most readily preventable HA-BSIs. Multimodal intervention programmes in ICUs virtually eliminated central venous catheterrelated HA-BSIs in one study. ${ }^{24}$ Clear guidelines exist about minimising the risk of IVC-related HA-BSI, and the extent to which they are followed can be evaluated by reviewing the medical chart. For example, an HA-BSI originating from a peripheral venous catheter left in place for longer than $96 \mathrm{~h}$ in a patient without difficult venous access was considered certainly preventable (box 1) ${ }^{10}$ By contrast, the use of measures to prevent other forms of infection (eg, aseptic handling of urinary devices or skin preparation before surgery) may be more difficult to evaluate. It was therefore difficult to determine whether preventive measures were observed in these cases, and evaluation of preventability was partly based on presence of underlying diseases and risk factors in the bacteraemic patients, and whether these infections are considered preventable in the literature.

The mortality from HA-BSI was high in our population. We were unable to determine the contribution to this high mortality of factors related to the HA-BSIs and of factors related to underlying diseases. High mortalities have been reported in patients with HA-BSI and underlying diseases associated with poor life expectancy. ${ }^{2}$
Our study has a number of limitations. First, whereas a role for healthcare is easier to determine for HA-BSIs than for other infections, preventability is difficult to assess. Although evidence in the literature can be used to develop criteria for preventability, the assessment relies in part on clinical judgement. The impact of this factor was probably limited in our study, since the investigators were trained hospital epidemiologists who were specialised in infection control. Nevertheless, review of the cases by an independent panel of experts might have increased the reliability of the data. Second, reviewing cases with attending physicians may miss errors, opportunities for prevention and lapses in organisation. Furthermore, the standard of practice that forms the basis for such judgement is often not precisely defined, and thus it may be susceptible to variation in interpretation. Indeed, it is recommended that all the members of the healthcare staff usually participate in morbidity and mortality reviews. However, our method required only about $45 \mathrm{~min}$ for each case. It may be useful for identifying wards or infections that require special attention. Third, $24.6 \%$ of all HA-BSIs were considered of uncertain preventability. This high proportion underlines the difficulty of preventability assessments. A more detailed review would probably identify a higher proportion of preventable HABSIs. Moreover, a blinded independent review would have increased the validity of the assessed preventability. Finally, our study was conducted in a single centre, and the investigators were physicians at that centre. Therefore, our results may not apply to other hospitals.

In conclusion, $56 \%$ of HA-BSIs in our hospital were not preventable, whereas $19.4 \%$ were certainly or probably preventable, most of them being IVC-related infections. These preliminary results require confirmation in a multicentre study with an external assessment of preventability. If validated, the simple method used in our study might prove useful for identifying infections that require a detailed review, and to bring to public attention that HAIs may not be preventable in every instance.

\section{Competing interests None.}

Ethics approval Ethics approval was provided by the Institutional Review Board of the Bichat Claude-Bernard Hospital.

Provenance and peer review Not commissioned; externally peer reviewed.

\section{REFERENCES}

1. Haley RW, Culver DH, White JW, et al. The efficacy of infection surveillance and control programs in preventing nosocomial infections in US hospitals. Am J Epidemiol 1985; 121:182-205

2. Pittet D, Li N, Woolson RF, et al. Microbiological factors influencing the outcome of nosocomial bloodstream infections: a 6-year validated, population-based model. Clin Infect Dis 1997;24:1068-78.

3. Pittet D, Tarara D, Wenzel RP. Nosocomial bloodstream infection in critically ill patients. Excess length of stay, extra costs, and attributable mortality. JAMA 1994;271:1598-601.

4. Renaud B, Brun-Buisson C. Outcomes of primary and catheter-related bacteremia. A cohort and case-control study in critically ill patients. Am J Respir Crit Care Med 2001;163:1584-90. 
5. Brennan TA, Leape LL, Laird NM, et al. Incidence of adverse events and negligence in hospitalized patients. Results of the Harvard Medical Practice Study I. N Engl J Med 1991;324:370-6

6. Harbarth S, Sax H, Gastmeier P. The preventable proportion of nosocomial infections: an overview of published reports. J Hosp Infect 2003;54:258-66.

7. Vincent C, Neale G, Woloshynowych M. Adverse events in British hospitals: preliminary retrospective record review. BMJ 2001;322:517-19.

8. Michel P, Quenon JL, Djihoud A, et al. French national survey of inpatient adverse events prospectively assessed with ward staff. Qual Saf Health Care 2007:16:369-77.

9. Wenzel RP, Edmond MB. The impact of hospital-acquired bloodstream infections. Emerg Infect Dis 2001;7:174-7.

10. Centers for Disease Control and Prevention. Guidelines for the prevention of intravascular catheter-related infections. MMWR 2002;51(No. RR-10):1-29.

11. Garner JS, Jarvis WR, Emori TG, et al. CDC definitions for nosocomial infections, 1988. Am J Infect Control 1988;16:128-40.

12. Brun-Buisson C, Abrouk F, Legrand P, et al. Diagnosis of central venous catheter-related sepsis. Critical level of quantitative tip cultures. Arch Intern Med 1987:147:873-7.

13. Blot F, Nitenberg G, Chachaty E, et al. Diagnosis of catheter-related bacteraemia: a prospective comparison of the time to positivity of hub-blood versus peripheralblood cultures. Lancet 1999;354:1071-7.

14. McCabe WR, Jackson GG. Gram-negative bacteremia. II. Etiology and ecology. Ann Intern Med 1962:110:847-55.

15. Hayward RA, Hofer TP. Estimating hospital deaths due to medical errors: preventability is in the eye of the reviewer. JAMA 2001;286:415-20.
16. Edmond M, Eickhoff TC. Who is steering the ship? External influences on infection control programs. Clin Infect Dis 2008;46:1746-50.

17. Michel P, Quenon JL, de Sarasqueta AM, et al. Comparison of three methods for estimating rates of adverse events and rates of preventable adverse events in acute care hospitals. BMJ 2004;328:199.

18. Pittet D, Wenzel RP. Nosocomial bloodstream infections. Secular trends in rates mortality, and contribution to total hospital deaths. Arch Intern Med 1995; 155:1177-84

19. Pittet D. Nosocomial bloodstream infections. In: Wenzel RP, ed. Prevention and control of nococomial infections. 3rd edn. Baltimore, MD: Williams \& Wilkins, 1997:711-69.

20. Andersen BM, Ringertz SH, Gullord TP, et al. A three-year survey of nosocomial and community-acquired infections, antibiotic treatment and re-hospitalization in a Norwegian health region. J Hosp Infect 2000;44:214-23.

21. Delgado-Rodriguez $\mathbf{M}$, Gomez-Ortega A, Sillero-Arenas M, et al. Efficacy of surveillance in nosocomial infection control in a surgical service. Am J Infect Control 2001:29:289-94.

22. Gastmeier $\mathbf{P}$, Brauer $\mathrm{H}$, Forster $\mathrm{D}$, et al. A quality management project in 8 selected hospitals to reduce nosocomial infections: a prospective, controlled study. Infect Control Hosp Epidemiol 2002:23:91-7.

23. Rossello-Urgell J, Vaque-Rafart J, Hermosilla-Perez E, et al. An approach to the study of potentially preventable nosocomial infections. Infect Control Hosp Epidemio 2004;25:41-6.

24. Berenholtz SM, Pronovost PJ, Lipsett PA, et al. Eliminating catheter-related bloodstream infections in the intensive care unit. Crit Care Med 2004:32:2014-20. 